\title{
Validation of the French version of the Wisconsin Quality of Life (WISQOL) questionnaire for patients with nephrolithiasis
}

Naeem Bhojani ${ }^{1}$; Ghizlane Moussaoui ${ }^{2}$; David-Dan Nguyen ${ }^{1}$; Mei Juan Trudel ${ }^{1}$; Garo-Shant Topouzian ${ }^{1}$; Nare-Gacia Topouzian ${ }^{1}$; Kristina L. Penniston ${ }^{3}$; Sero Andonian ${ }^{2}$

${ }^{1}$ Division of Urology, University of Montreal, Montreal, QC, Canada; ${ }^{2}$ Division of Urology, McGill University, Montreal, QC, Canada; ${ }^{3}$ Department of Urology, University of Wisconsin, Madison, WI, United States

Acknowledgements: Ghizlane Moussaoui was the recipient of a Summer Research Bursary.

Cite as: Bhojani N, Moussaoui G, Nguyen D-D, et al. Validation of the French version of the Wisconsin Quality of Life (WISQOL) questionnaire for patients with nephrolithiasis. Can Urol Assoc J 2020 September 28; Epub ahead of print. http://dx.doi.org/10.5489/cuaj.6552

Published online September 28, 2020

$* * *$

\section{Abstract}

Introduction: The Wisconsin Stone Quality of Life (WISQOL) questionnaire has been recently developed to objectively assess QOL in patients with nephrolithiasis. However, a French version of the questionnaire was lacking. Therefore, the aim of the present study was to develop and validate the French version of this tool.

Methods: The French version of the WISQOL (F-WISQOL) was developed in a multistep process involving primary translation, back-translation, and pilot testing among a group of patients $(n=12)$. Nephrolithiasis patients from two tertiary care institutions were recruited into this study and completed the following questionnaires: the medical history form and either the WISQOL or F-WISQOL. Internal consistency was assessed using Cronbach's $\alpha$, and interdomain associations were evaluated using Spearman's rank correlation (r). One-way ANOVA was used to compare scores from the two groups (WISQOL and F-WISQOL).

Results: A total of 210 patients were enrolled in this study: 68 in the WISQOL group and 148 in the F-WISQOL group. Internal consistency was high for all domains in both groups (FWISQOL: 0.924-0.970; WISQOL: 0.888-0.965). No statistically significant difference was found between the two groups' scores. Inter-domain association, measured by Spearman correlation, was moderate to very strong between all the domains in the F-WISQOL. Values ranged from $\mathrm{r}=0.676-0.915$, with acceptable correlation between $\mathrm{D} 1$, D2, and D3, but weaker correlation between D4 (vitality) and the three other domains ( $\mathrm{r}=0.676-0.729)$. 
Conclusions: In the present study, the French version of the WISQOL questionnaire was validated at two academic institutions.

\section{Introduction}

With recurrence rates of up to $50 \%$ at 5 years post a symptomatic kidney stone event, kidney stone disease is often a chronic medical condition that poses a significant burden on Quality of Life (QOL). ${ }^{1}$ Previous studies exploring the QOL of nephrolithiasis patients found pain and physical impairment to be worse in this patient population compared to their healthy counterparts. ${ }^{2}$ Mental health symptoms such as anxiety and depression were also reported to be worse amongst recurrent nephrolithiasis patients during their inter crises intervals. ${ }^{3,4}$ The number of recurrent colic episodes significantly correlated with symptoms of anxiety $(\mathrm{P}=0.002)$ and depression $(\mathrm{P}<0.001) .{ }^{3}$ While current guidelines recommend treatment options based on stonefree rates, patients' QOL is often not included as a primary end point. ${ }^{5}$

In recent years, Health-related quality of life (HRQOL) data has gained more attention from clinicians and researchers as it has an important role in disease management. HRQOL scores are thought to provide critical information regarding patients' expectations of their health state post-treatment, which may impact decision-making. This is particularly relevant in the management of asymptomatic stones, where information about the patients' expectations of HRQOL improvement could influence the choice between active management versus surveillance. ${ }^{6}$ Consideration of HRQOL factors also provides vital information that allow for medical and nutritional therapies to be tailored to patients and their treatment goals. One such example in urologic oncology is the incorporation of the Bladder Utility Symptoms Scale in the management of patients with prostate and bladder cancers. ${ }^{7,8,9}$

Another example of HRQOL questionnaire is the Ureteral Stent Symptom Questionnaire (USSQ) that was previously developed and validated to assess QOL in patients with ureteral stents. ${ }^{10}$ One of the shortcomings of the USSQ is that the majority of the items assessed HRQOL specific to patients with ureteral stents. The Wisconsin Stone-QOL (WISQOL) self-report questionnaire was developed in 2016 to address this gap in the literature and clinical practice. ${ }^{11}$ Although general measures of QOL are important, they are often insufficient to gain a holistic and comprehensive understanding of the disease burden specifically experienced by nephrolithiasis patients. The WISQOL questionnaire's objective is to measure patients' QOL in the context of their disease as well as to promote and incorporate this new tool within current clinical management paradigms for nephrolithiasis. It has been previously established, through internal validity testing, that the WISQOL questionnaire successfully discriminates between patients on the basis of nephrolithiasis. ${ }^{6}$ Although, the generalizability of this tool to patients of a broader geographical area has been previously assessed in Turkey, Spain and Korea, the 
questionnaire had yet to be tailored to the French-speaking population in Canada and beyond. ${ }^{12,13,14}$ The purpose of this study was to develop the French version of the WISQOL (FWISQOL) and evaluate its internal consistency and psychometric properties.

\section{Methods}

\section{Translation method and pilot testing}

The French version of the questionnaire (F-WISQOL; Appendix A) was developed in a multistep process by two authors who are both bilingual with native level written and spoken French and English. Both authors were medical students with knowledge in medical jargon in both languages. ${ }^{15}$ The questionnaire was first translated from English to French by one of the authors (GST) and subsequently back translated from French to English by another author (NGT). The back translated version found to be consistent with the original WISQOL questionnaire, except for one question that was revised in the F-WISQOL. A final review of the latest version of the questionnaire was conducted by an endourologist (SA) to ensure the F-WISQOL was representative of the original version and that it did not include vocabulary that would be difficult for patients to understand. This second version of the F-WISQOL was then pilot tested amongst a group of 12 nephrolithiasis patients recruited in the outpatient clinic. All patients included in the pilot testing completed the second version of the F-WISQOL questionnaire in its entirety and did not raise any issues in understanding the meaning of any items. Some grammatical and punctuation adjustments were made at that point. The revised third version was used for the validation of the F-WISQOL. Final correction of minor grammatical errors took place during the review of this manuscript.

\section{Sample size calculation}

In previous translation studies, the number of patients varied between 5 and $87 .{ }^{12,13,14} \mathrm{~A}$ correlation sample size calculation was performed using the Sample Size Calculators for designing clinical research tool (UCSF Clinical \& Translational Science Institute) ${ }^{16}$. The calculation yielded that recruiting a total of 194 patients would be sufficient; using $\alpha$ (two-tailed) $=0.05, \beta=0.20$ and $r=0.20$. The goal for the present study was to recruit at least 100 patients at each of the two tertiary care centres.

\section{Study population}

Ethics board approval was obtained from both institutions and all patients were consented prior to enrolment. Adult males and females with a history of kidney stones and proficient in either English or French were approached for the study. Exclusion criteria included: illiteracy, inability to communicate in English or French, as well as severe mental or physical disability preventing informed consent and/or independent questionnaire completion. Patients with common urological comorbidities such as neurogenic bladder or benign prostatic hyperplasia were not 
excluded from the study, since they are at increased risk of developing nephrolithiasis. ${ }^{17,18}$ Both symptomatic and asymptomatic patients were recruited into the study.

A total of 106 adult patients with nephrolithiasis were recruited from the outpatient stone clinic of the McGill University Health Centre (MUHC, Glen site), an academic tertiary-care centre affiliated with McGill University. At the MUHC, 115 patients were approached making the enrollment ratio $92.2 \%$. Another 104 patients with nephrolithiasis were recruited from the Centre Hospitalier de l'Université de Montréal (CHUM), an academic tertiary-care centre affiliated with the University of Montréal. At the CHUM, 114 patients were approached making the enrollment ratio $91.2 \%$. Both centers provide universal outpatient and inpatient health coverage in their respective catchment areas to the greater Montreal area. Furthermore, both tertiary care centres provide the same types of surgeries to patients with nephrolithiasis. The recruitment period spanned between July 2018 and April 2019.

\section{Study design}

Enrolled patients were asked to complete either the WISQOL or F-WISQOL questionnaire based on their preference depending on whether they felt most comfortable with English or French, respectively. In addition, all patients were asked to fill a medical history form specifically developed for this study. The latter questionnaire was translated into French for those more comfortable in French. The WISQOL questionnaire is a 28 -item multidimensional questionnaire measuring morbidity in four domains specific to nephrolithiasis patients: social impact, emotional impact, disease impact and impact on vitality. ${ }^{6}$ Each item in the questionnaire is scored on a Likert-scale ranging from 1 to 5, with the maximum score being of 140. A higher score in the WISQOL indicates a higher HRQOL. The French version of the WISQOL (FWISQOL) was constructed in the same format. The WISQOL only refers to events or symptoms occurring in the past 4 weeks. The medical history form aimed to gather information about the patients' primary occupation, medical and nephrolithiasis history and current pharmacological therapies. The questionnaires included short instructions and patients were encouraged to ask clarifying questions to the research assistant prior to beginning. Upon completion, patients were asked if they had any comments or suggestions regarding the WISQOL items.

The primary aim of this study was to develop and validate F-WISQOL. To achieve this, assessment of the internal consistency within each domain and inter-domain was performed for the F-WISQOL questionnaire. The secondary outcome was to compare the internal consistency between the French (F-WISQOL) and the previously validated English version of the WISQOL questionnaire. Both primary and secondary outcomes were assessed by means of statistical analyses.

Statistical analysis was completed using IBM SPSS Statistics version 24.0 (IBM Corp., Chicago, USA). For the purpose of the analyses, raw scores and totals were used. The level of significance was set at $p<0.05$. Consistency and reliability were assumed at values $>0.70$. Baseline demographics and clinical characteristics were compared between both groups (French 
and English). Student t-tests and Chi-square analyses were used as appropriate. For the primary outcome, inter-domain associations were measured using Spearman's rank correlation ( $\mathrm{r}$ ). Principal component analysis with Kaiser-Varimax rotation was done to identify the most important variables of the French version of the WISQOL and to group them into the most important components (domains). For the secondary outcome, internal consistency reliability was assessed using Cronbach's $\alpha$, comparing the two versions of the questionnaire across all domains. Readability was assessed using the Flesch-Kincaid test. ${ }^{19}$

\section{Results}

All patients enrolled $(n=210)$ completed either the WISQOL or F-WISQOL questionnaire. At the MUHC, a total of 106 participants were recruited. Of those, $36 \%(n=38)$ completed the French version of the questionnaire, while $64 \%(n=68)$ completed the English version of the questionnaire. At the CHUM, which cares for a predominantly francophone population, 100\% $(n=104)$ of the recruited participants completed the F-WISQOL. Therefore, the French group was comprised of 142 patients whereas 68 patients were in the English group. Both WISQOL and FWISQOL groups were comparable except for two variables: there were significantly more women who took the WISQOL questionnaire than the F-WISQOL questionnaire (50\% vs. 35\%; $\mathrm{p}=0.032$ ). In addition, there were significantly more patients with history of prior stone passage in the WISQOL group than in the F-WISQOL group (67.7\% vs 50\%; $\mathrm{p}=0.016)$ (Table 1$)$.

On one-way analysis of variance (ANOVA), there were no significant differences in the WISQOL and F-WISQOL groups (Table 2). Cronbach's $\alpha$ was used to assess the internal consistency within each domain of the WISQOL and F-WISQOL questionnaires (Table 3). Internal consistency was high for all domains for both questionnaires ranging from 0.89 to 0.97 for the WISQOL and from 0.92 to 0.97 for the F-WISQOL (Table 3). Table 4 illustrates interdomain association in the F-WISQOL, measured by Spearman correlation. Moderate to very strong associations were found between all of the domains. Values ranged from $\mathrm{r}=0.68-0.91$, with acceptable correlation between D1, D2 and D3, but weaker correlation between D4 (vitality) and the 3 other domains $\mathrm{r}=0.68-0.73$.

Principal components analysis generated Eigenvalues indicating that 4 factors (domains) accounted for nearly $70 \%$ of cumulative variability, which is consistent with the number identified in the original development of the WISQOL. After Kaiser-Varimax rotation, none of the 28 items of the F-WISQOL loaded $<0.30$ on a factor, and exploratory factor analysis revealed individual items had high communalities ranging between 0.48 and 0.87 for all 28 items (mean 0.73; median 0.72). Flesch-Kincaid score was of 10, indicating the F-WISQOL's readability is at the level of $10^{\text {th }}$ grade. 


\section{Discussion}

Kidney stone disease is a chronic condition that significantly affect patients' HRQOL. Patients with recurrent nephrolithiasis report depression, lower general health scores and increased bodily pain. ${ }^{20,21}$ Although previous studies have assessed QOL in kidney stone patients using general physical and mental health questionnaires, a gap remained in the literature for a disease-specific assessment tool. The WISQOL was created in response to this call. In this study, the French version of the validated WISQOL (F-WISQOL) self-reported questionnaire was developed and validated to accommodate the needs of French-speaking patient populations.

The results of this study demonstrate that the F-WISQOL is a valid assessment tool for the evaluation of nephrolithiasis patients' symptoms. Statistical analyses yielded no categorical differences between responses on the WISQOL and F-WISQOL versions. With all patients being from similar backgrounds and institutions, our data suggest that the instruments performed the same.

Cronbach's alpha was high across all domains in the French version (Cronbach's $\alpha=$ 0.97), suggesting that the items are highly correlated. Similar results were found with the original English version (WISQOL) suggesting high internal consistency of the translated (F-WISQOL) version. ${ }^{11}$ Although coefficients greater than 0.95 can be suggestive of redundancy, exploratory factor analysis demonstrated high communalities across all items suggesting that there are no items that stand out as being irrelevant or unnecessary to the questionnaire.

Inter-domain reliability analysis showed strong correlations between all 4 of the domains comprised in the F-WISQOL. This is indicative of the ability of the different domains to capture HRQOL effects in a population of nephrolithiasis patients. These findings are in accordance with the results of the original WISQOL study and the more recent Turkish validity study. ${ }^{11,12} \mathrm{~A}$ moderate correlation was found between the vitality domain and D2 and D3, which was also reported in the original WISQOL paper. However, it remains uncertain why this latter correlation is not as strong as the other ones. ${ }^{11}$ This association is interesting as it could suggest patients with lower HRQOL in the context of nephrolithiasis also tend to experience higher levels of stress.

This study is not without limitations. Due to lack of financial support for this project, two independent professional translators were not used to translate the original WISQOL to the FWISQOL. Instead, two medical students with native level written and spoken French and English languages translated the original WISQOL to F-WISQOL. While the translation process deviated from the currently accepted guidelines, the advantage of using health care professionals for the translation process include their understanding of medical jargon in both French and English. Thus, the final revised version of the F-WISQOL presented in this article represents a comprehensible and user-friendly questionnaire. For logistical reasons, follow-up questionnaires could not be sent out to patients, which made impossible a test-retest reliability analysis. Convergent reliability was also not assessed because our methodology did not include the completion of a separate questionnaire evaluating general health domains. Nevertheless, in the 
present study, the French version of the original WISQOL was developed and validated in two tertiary care university hospitals in Canada.

\section{Conclusions}

This multicenter study demonstrated that the French version of the WISQOL (F-WISQOL) is a reliable instrument that can be used in clinical settings to evaluates the QOL in French-speaking patients with kidney stones. The items appear to measure the same underlying construct as the original English version. Further use of the questionnaire in clinical practice will help to identify clinically relevant differences between patients and within a patient when there are changes in their stone disease and/or symptom status over time. 


\section{References}

1. Khan SR, Pearle MS, Robertson WG, Gambaro G, Canales BK, Doizi S, et al. Kidney stones. Nat Rev Dis Primer. 2016 Dec;2(1):16008.

2. Patel N, Brown RD, Sarkissian C, De S, Monga M. Quality of life and urolithiasis: the patient - reported outcomes measurement information system (PROMIS). Int Braz J Urol. 2017 Oct;43(5):880-6.

3. Diniz DHMP, Blay SL, Schor N. Anxiety and depression symptoms in recurrent painful renal lithiasis colic. Braz J Med Biol Res. 2007 Jul;40(7):949-55.

4. Diniz DHMP, Schor N, Blay SL. Stressful Life Events and Painful Recurrent Colic of Renal Lithiasis. J Urol. 2006 Dec;176(6):2483-7.

5. Ordon M, Andonian S, Blew B, Schuler T, Chew B, Pace KT. CUA Guideline: Management of ureteral calculi. Can Urol Assoc J. 2015 Dec 14;9(11-12):837.

6. Penniston KL, Nakada SY. Development of an Instrument to Assess the Health Related Quality of Life of Kidney Stone Formers. J Urol. 2013 Mar;189(3):921-30.

7. Perlis N, Krahn MD, Boehme KE, Alibhai SMH, Jamal M, Finelli A, et al. The Bladder Utility Symptom Scale: A Novel Patient Reported Outcome Instrument for Bladder Cancer. J Urol. 2018 Aug;200(2):283-91.

8. Feuerstein MA, Jacobs M, Piciocchi A, Bochner B, Pusic A, Fayers P, et al. Quality of life and symptom assessment in randomized clinical trials of bladder cancer: A systematic review. Urol Oncol Semin Orig Investig. 2015 Jul;33(7):331.e17-331.e23.

9. Kim SP, Karnes RJ, Nguyen PL, Ziegenfuss JY, Han LC, Thompson RH, et al. Clinical Implementation of Quality of Life Instruments and Prediction Tools for Localized

Prostate Cancer: Results from a National Survey of Radiation Oncologists and Urologists. J Urol. 2013 Jun;189(6):2092-8.

10. Joshi HB, Newns N, Stainthorpe A, MacDonagh RP, Keeley FX, Timoney AG. Ureteral stent symptom questionnaire: development and validation of a multidimensional quality of life measure. J Urol. 2003 Mar;169(3):1060-4.

11. Penniston KL, Antonelli JA, Viprakasit DP, Averch TD, Sivalingam S, Sur RL, et al. Validation and Reliability of the Wisconsin Stone Quality of Life Questionnaire. J Urol. 2017 May;197(5):1280-8.

12. Atalay HA, Ülker V, Canat L, Özer M, Can O, Penniston KL. Validation of the Turkish version of the Wisconsin stone-quality of life questionnaire. Turk J Urol. 2019;45(2):118-23.

13. Basulto-Martínez M, Olvera-Posada D, Velueta-Martínez IA, Méndez-Probst C, FloresTapia JP, Penniston K, et al. Quality of life in patients with kidney stones: translation and validation of the Spanish Wisconsin Stone Quality of Life Questionnaire. Urolithiasis. 2020 May 5;

14. Yoon YE, Cho SY. Translation and Linguistic Validation of the Korean Version of the Wisconsin Stone Quality of Life Questionnaire. Int Neurourol J. 2020 Mar;24(1):77-83.

15. Eremenco SL, Cella D, Arnold BJ. A Comprehensive Method for the Translation and Cross-Cultural Validation of Health Status Questionnaires. Eval Health Prof. 2005 Jun;28(2):212-32. 
16. Khon M, Senyak J. Sample Size Calculators [Internet]. UCSF CTSI. 2020 [cited 2020 Jul 12]. Available from: https://www.sample-size.net

17. Stephany HA, Clayton DB, Tanaka ST, Thomas JC, Pope JC, Brock JW, et al. Development of upper tract stones in patients with congenital neurogenic bladder. $\mathrm{J}$ Pediatr Urol. 2014 Feb;10(1):112-7.

18. Huang W, Cao J-J, Cao M, Wu H-S, Yang Y-Y, Xu Z-M, et al. Risk factors for bladder calculi in patients with benign prostatic hyperplasia. Medicine (Baltimore). 2017 Aug;96(32):e7728.

19. Flesch R. A new readability yardstick. J Appl Psychol. 1948 Jun;32(3):221-33.

20. Angell J, Bryant M, Tu H, Goodman M, Pattaras J, Ogan K. Association of Depression and Urolithiasis. Urology. 2012 Mar;79(3):518-25.

21. Bryant M, Angell J, Tu H, Goodman M, Pattaras J, Ogan K. Health Related Quality of Life for Stone Formers. J Urol. 2012 Aug;188(2):436-40. 
Figures and Tables

\begin{tabular}{|l|c|c|c|}
\hline \multicolumn{4}{|c|}{ Table 1. Demographic and clinical characteristics } \\
\hline & $\begin{array}{c}\text { WISQOL } \\
\mathbf{n = 6 8}\end{array}$ & $\begin{array}{c}\text { F-WISQOL } \\
\mathbf{n = 1 4 2}\end{array}$ & Statistics \\
\hline Mean age (years) & $56.0+/-13.1$ & $53.2+/-14.0$ & $\mathrm{t}(208)=1.401, \mathrm{p}=0.163$ \\
\hline Age range(years) & $21.0+/-84.1$ & $18.9+/-79.6$ & $\mathrm{~N} / \mathrm{A}$ \\
\hline $\begin{array}{l}\text { Age of onset } \\
\text { (years) }\end{array}$ & $38.7+/-17.1$ & $39.2+/-15.3$ & $\mathrm{t}(186)=0.226, \mathrm{p}=0.822$ \\
\hline Female & $34(50.0 \%)$ & $49(34.5 \%)$ & $\mathrm{X}^{2}(1)=4.617, \mathbf{p}=\mathbf{0 . 0 3 2}$ \\
\hline BMI & $27.0+/-5.7$ & $27.9+/-5.7$ & $\mathrm{t}(177)=0.988, \mathrm{p}=0.324$ \\
\hline $\begin{array}{l}\text { Number of } \\
\text { comorbidities }\end{array}$ & $1.3+/-1.9$ & $1.5+/-1.5$ & $\mathrm{t}(205)=0.631, \mathrm{p}=0.529$ \\
\hline $\begin{array}{l}\text { History of prior } \\
\text { surgery }\end{array}$ & $38(55.9 \%)$ & $91(64.1 \%)$ & $\mathrm{X}^{2}(1)=1.306, \mathrm{p}=0.253$ \\
\hline $\begin{array}{l}\text { History of prior } \\
\text { stone passage }\end{array}$ & $46(67.6 \%)$ & $71(50.0 \%)$ & $\mathrm{X}^{2}(1)=5.804, \mathbf{p}=\mathbf{0 . 0 1 6}$ \\
\hline $\begin{array}{l}\text { Currently has } \\
\text { stones }\end{array}$ & $31(45.6 \%)$ & $61(43.0 \%)$ & $\mathrm{X}^{2}(3)=3.063, \mathrm{p}=0.382$ \\
\hline $\begin{array}{l}\text { Currently has stone } \\
\text { related symptoms }\end{array}$ & $22(32.4 \%)$ & $42(29.6 \%)$ & $\mathrm{X}^{2}(3)=1.452, \mathrm{p}=0.693$ \\
\hline
\end{tabular}

BMI: body mass index; WISQOL: Wisconsin Stone Quality of Life.

\begin{tabular}{|c|c|c|c|}
\hline & WISQOL & F-WISQOL & $\begin{array}{c}\text { Statistics - } \\
\text { ANOVA }\end{array}$ \\
\hline Domains & Mean \pm SD & Mean \pm SD & \\
\hline Social impact & $82.6 \pm 22.8$ & $76.5 \pm 28$ & $\begin{array}{c}F(1,196)=2.35, \\
p=0.13\end{array}$ \\
\hline Emotional impact & $74.6 \pm 28.9$ & $68.1 \pm 29$ & $\begin{array}{c}\mathrm{F}(1,196)=2.16 \\
\mathrm{p}=0.14\end{array}$ \\
\hline Disease impact & $72.7 \pm 25.9$ & $69.3 \pm 27.6$ & $\begin{array}{c}\mathrm{F}(1,196)=0.70 \\
\mathrm{p}=0.40\end{array}$ \\
\hline Impact on vitality & $65.8 \pm 31.2$ & $64.4 \pm 34.1$ & $\begin{array}{c}\mathrm{F}(1,196) 0.08 \\
\mathrm{p}=0.78\end{array}$ \\
\hline $\begin{array}{l}\text { Total standardized } \\
\text { score }\end{array}$ & $75.9 \pm 22.4$ & $71.5 \pm 25.0$ & $\begin{array}{c}F(1,196)=1.40, \\
p=0.24\end{array}$ \\
\hline
\end{tabular}

SD: standard deviation; WISQOL: Wisconsin Stone Quality of Life. 


\begin{tabular}{|l|c|c|}
\hline \multicolumn{2}{|l|}{$\begin{array}{l}\text { Table 3. Internal Consistency of WISQOL French and English } \\
\text { versions (Cronbach's } \boldsymbol{\alpha} \text { ) }\end{array}$} & $\begin{array}{l}\text { Internal consistency - Cronbach's } \boldsymbol{\alpha} \\
\text { WISQOL } \\
\text { F-WISQOL }\end{array}$ \\
\hline Domains & \multicolumn{2}{|c|}{} \\
\hline Social impact & 0.914 & 0.924 \\
\hline Emotional impact & 0.956 & 0.940 \\
\hline Disease impact & 0.915 & 0.924 \\
\hline Impact on vitality & 0.888 & 0.925 \\
\hline All domains & 0.966 & 0.970 \\
\hline
\end{tabular}

WISQOL: Wisconsin Stone Quality of Life.

\begin{tabular}{|c|c|c|c|c|c|}
\hline Domains & & D1 & D2 & D3 & D4 \\
\hline Social impact D1 & $\begin{array}{l}\mathrm{r} \\
\mathrm{p}\end{array}$ & $\begin{array}{l}- \\
-\end{array}$ & & & \\
\hline Emotional impact D2 & $\begin{array}{l}\mathrm{r} \\
\mathrm{p}\end{array}$ & $\begin{array}{c}0.816 \\
p<0.001\end{array}$ & & & \\
\hline Disease impact D3 & $\begin{array}{l}\mathrm{r} \\
\mathrm{p}\end{array}$ & $\begin{array}{c}0.775 \\
\mathrm{p}<0.001\end{array}$ & $\begin{array}{c}0.752 \\
p<0.001\end{array}$ & & \\
\hline Impact on vitalityD4 & $\begin{array}{l}\mathrm{r} \\
\mathrm{p}\end{array}$ & $\begin{array}{c}0.676 \\
p<0.001\end{array}$ & $\begin{array}{c}0.678 \\
p<0.001\end{array}$ & $\begin{array}{c}0.729 \\
p=0.001\end{array}$ & \\
\hline All domains & $\mathrm{r}$ & $\begin{array}{c}0.906 \\
p<0.001\end{array}$ & $\begin{array}{c}0.910 \\
\mathrm{p}<0.001\end{array}$ & $\begin{array}{c}0.915 \\
\mathrm{p}<0.001\end{array}$ & $\begin{array}{c}0.822 \\
\mathrm{p}<0.001\end{array}$ \\
\hline
\end{tabular}

WISQOL: Wisconsin Stone Quality of Life. 